\title{
Comparative Assessment and Outlook on Methods for Imputing Proteomics Data
}

\section{Minjie Shen}

Virginia Polytechnic Institute and State University

Yi-Tan Chang

Virginia Polytechnic Institute and State University

Chiung-Ting Wu

Virginia Polytechnic Institute and State University

\section{Sarah J Parker}

Cedars Sinai Medical Center

\section{Georgia Saylor}

Wake Forest University

\section{Yizhi Wang}

Virginia Polytechnic Institute and State University

\section{Guoqiang Yu}

Virginia Polytechnic Institute and State University

Jennifer E. Van Eyk

Cedars Sinai Medical Center

\section{Robert Clarke}

University of Minnesota

David M. Herrington

Wake Forest University

Yue Wang ( $\nabla$ yuewang@vt.edu )

Virginia Polytechnic Institute and State University

\section{Research Article}

Keywords: quantitative proteomics data analysis, evaluation methodologies, low-rank matrix factorization framework

Posted Date: March 18th, 2021

DOl: https://doi.org/10.21203/rs.3.rs-298864/v1

License: (9) (1) This work is licensed under a Creative Commons Attribution 4.0 International License. 



\section{Comparative assessment and outlook on methods for}

\section{2 imputing proteomics data}

4 Minjie Shen ${ }^{1, *}$, Yi-Tan Chang ${ }^{1, *}$, Chiung-Ting Wu ${ }^{1, *}$, Sarah J Parker ${ }^{2}$, Georgia

5 Saylor $^{3}$, Yizhi Wang ${ }^{1}$, Guoqiang Yu ${ }^{1}$, Jennifer E. Van Eyk ${ }^{2}$, Robert Clarke ${ }^{4}$,

6 David M. Herrington ${ }^{3}$, and Yue Wang ${ }^{1, \dagger}$

$7 \quad{ }^{*}$ Equal contribution; ${ }^{\dagger}$ Author for correspondence

$8 \quad{ }^{1}$ Department of Electrical and Computer Engineering, Virginia Polytechnic Institute and State

9 University, Arlington, VA 22203, USA; ${ }^{2}$ Advanced Clinical Biosystems Research Institute, Cedars

10 Sinai Medical Center, Los Angeles, CA 90048, USA; ${ }^{3}$ Department of Internal Medicine, Wake Forest

11 University, Winston-Salem, NC 27157, USA; ${ }^{4}$ The Hormel Institute, University of Minnesota, Austin,

12 MN 55912, USA

14 Abstract

15 Background: Missing values are a major issue in quantitative proteomics data

16 analysis. While many methods have been developed for imputing missing values in

17 high-throughput proteomics data, comparative assessment on the accuracy of

18 existing methods remains inconclusive, mainly because the true missing

19 mechanisms are complex and the existing evaluation methodologies are imperfect.

20 Moreover, few studies have provided an outlook of current and future development.

21 Results: We first report an assessment of eight representative methods collectively

22 targeting three typical missing mechanisms. The selected methods are compared on

23 both realistic simulation and real proteomics datasets, and the performance is

24 evaluated using three quantitative measures. We then discuss fused regularization

25 matrix factorization, a popular low-rank matrix factorization framework with similarity 
and/or biological regularization, which is extendable to integrating multi-omics data such as gene expressions or clinical variables. We further explore the potential application of convex analysis of mixtures, a biologically-inspired latent variable modeling strategy, to missing value imputation. The preliminary results on proteomics data are provided together with an outlook into future development directions.

Conclusion: While a few winners emerged from our comparative assessment, datadriven evaluation of imputation methods is imperfect because performance is evaluated indirectly on artificial missing or masked values not authentic missing values. Imputation accuracy may vary with signal intensity. Fused regularization matrix factorization provides a possibility of incorporating external information. Convex analysis of mixtures presents a biologically plausible new approach.

\section{Background}

Liquid chromatography coupled to mass spectrometry (LC-MS) is a popular method for high-throughput identification and quantification of thousands of proteins in a single analysis $[1,2]$. The LC-MS signals can be displayed in a three-dimensional space with the mass-to-charge ratios, retention times and intensities for the observed peptides. However, this approach suffers from many missing values at the peptide or protein level, which significantly reduces the amount of quantifiable proteins with an average of $44 \%$ missing values [3-5].

While there are multiple causes for this missingness, three typical missing mechanisms are widely acknowledged. Low abundant proteins may be missing because their concentration is below the lower limit of detection (LLD); while poorly ionizing peptides may cause proteins to be Missing Not at Random (MNAR) [6]. However, missingness may also extend to mid- and even high-range intensities, statistically 
categorized into Missing at Random (MAR) and Missing Completely at Random (MCAR) [7]. MAR is actually missing conditionally at random given the observed, known covariates, or even unknown covariates. MCAR depends neither on observed nor on the missing data, thus the incomplete data are representative for the entire data. Practically, MAR and MNAR cannot be distinguished because by definition missing values are unknown [8]. More importantly, missing values in reality can originate from a mix of both known and unknown missing mechanisms [7, 9].

A common solution for missingness is to impute the missing values based on assumed missing mechanisms. But, this comes at the expense of potentially introducing profound change in the distribution of protein-level intensities, because most of existing methods are designed specifically for a single missing mechanism. This can have unpredictable effects on downstream differential analyses. Moreover, while many imputation methods have been adopted for imputing missing values in proteomics data, comparative evaluation on their relative performance remains largely inconclusive, and few studies provide an outlook addressing unresolved problems or future development directions $[4,9,10]$.

To gain first-hand insight into the strengths and limitations of both imputation methods and assessment designs, we conduct a collective assessment of eight representative methods involving three typical missing mechanisms in conjunction with authentic missing values. Compared on a set of realistic and preserving simulations derived from real proteomics data sets, the performance of the selected methods is measured by three criteria, root-mean-square error (RMSE), normalized root-meansquare error (NRMSE), and Sum of Ranks (SOR). There are several important observations from this comparison study. First, while imputation methods perform differentially under various missing mechanisms, algorithmic parameter settings, and 
preprocessing procedures, there are a few methods that consistently outperformed peer methods across a range of realistic simulation studies. Second, the quality of performance assessment depends on the efficacy of simulation designs and a more realistic simulation design should include authentic missing values and preserve original overall data distribution. Third, existing assessment methodology is imperfect in that performance is indirectly assessed on imputing either artificial or masked, but not authentic missing values (see Discussion section).

To explore a more integrative strategy for improving imputation performance, we discuss a low-rank matrix factorization framework with fused regularization on sparsity and similarity - Fused Regularization Matrix factorization (FRMF) [11-13], which can naturally integrate other-omics data such as gene expression or clinical variables. We also introduce a biologically-inspired latent variable modeling strategy Convex analysis of Mixtures (CAM) [13, 14], which performs data imputation using the original intensity data (before log-transformation). The preliminary results on real proteomics data are provided together with an outlook into future development directions.

\section{Results}

\section{Experimental design and protocol}

We selected eight representative methods for comparative assessment, based on their intended missing mechanism(s) and imputation principles, summarized in Figure 1. One method (Min/2) is devoted to MNAR (LLD) [7], two methods (swKNN and pwKNN) are tailored to MAR (local-similarity) [15], and five methods (Mean, PPCA, NIPALS, SVD, and SVT) are designed for MCAR/MAR (global-structure or low-rank matrix factorization) $[7,9,16-18]$. We then explored and tested several variants of 
100

FRMF and CAM, where local similarity information is obtained from baseline or other

101 data acquired from the same samples.

102

103

104 portion (no authentic missing value) of a real proteomics dataset, where artificial

105 missing values were introduced involving two typical missing mechanisms and used

106 for performance assessment. Second, the realistic simulation data were generated from

107 the complete data matrix (including authentic missing values) of a real proteomics

108 dataset, where a small percentage of data points were randomly set-aside (masked

109 values) and used solely for performance assessment. The preprocessing eliminates

110 those proteins whose missing rates are higher than $80 \%$ and then performs $\log 2$

111 transformation [19]. The parameters were optimized for each imputation method by

112 parameter sweeping over a wide range of settings at each missing rate. The overall

113 experimental workflow is given in Figure 2.

\section{Real proteomics data}

115 The real LC-MS proteomics data form the base from which the simulation data sets

116 were produced [6]. The data were acquired using data-independent acquisition (DIA)

117 protocol, and protein level output was generated by mapDIA [20]. The dataset contains

118200 samples associated with 2,682 proteins measured in human left anterior descending

119 (LAD) coronary arteries collected as part of a study of coronary and aortic

120 atherosclerosis [21]. The data were produced in three separate batches, indexed by A,

$121 \mathrm{~B}$, and $\mathrm{C}$, and all have passed quality control and preprocessing procedures,

122 summarized in Table 1 (Supplementary Information).

123 Table 1. Summary of real proteomics datasets used in this work. 


\begin{tabular}{lccccc}
\hline & Sample size & $\begin{array}{c}\text { Total Missing Rate } \\
\text { \#MV/(\#Sample*\#Protein) }\end{array}$ & $\begin{array}{c}\text { Setting \#1 protein } \\
\text { size } \\
\text { (non-missing proteins) }\end{array}$ & $\begin{array}{c}\text { Setting \#2 protein } \\
\text { (proteins with } \\
\text { missing rate) }\end{array}$ \\
\hline Batch A & 98 & 2107 & $24.67 \%$ & $751(35.64 \%)$ & $1935(91.84 \%)$ \\
Batch B & 55 & 2604 & $29.63 \%$ & $819(31.45 \%)$ & $2324(89.25 \%)$ \\
Batch C & 47 & 2590 & $25.52 \%$ & $976(37.68 \%)$ & $2325(89.77 \%)$ \\
\hline
\end{tabular}

125 Simulation data generated from the observed portion of data matrix

126 Based on the observed data portion (no authentic missing values), we adopted a hybrid

127 missing data model and used the R package imputeLCMD to introduce artificial

128 missing values while preserving the original observed data patterns [22]. Specifically,

129 MCAR missing values were introduced by randomly replacing some data points with

130 'NA' (not available) according to the designed missing rates (approximately from 1\%

131 to 50\%); MNAR missing values were introduced by quantile cut-off for the full data

132 set $[7,9,19]$; and mixed MCAR and MNAR missing values were introduced by

133 assigning $(1-\beta)$ portion of MCAR and $\beta$ portion of MNAR; corresponding to

134 missing rate $\alpha$ and $\beta=0,0.1,1$ (Supplementary Information).

\section{Simulation data with set-aside masked values from the full data matrix}

136 In this simulation setting, we used the full data matrix (including both observed and

137 authentic missing values) from the human coronary proteomics data set. To preserve

138 the original patterns of both observed and authentic missing data, for each protein, a

139 small percentage of data points in the complete data matrix were randomly set-aside as

140 'NA' (masked values) with the masking rate(s) proportional to the authentic missing

141 rate(s). This procedure was repeated for all proteins and the masked values were 
142 considered as a mix of MNAR and MAR conditioned on the observed missing rates

143 and data patterns (Figure 3, Supplementary Information).

\section{Performance assessment focused on MNAR}

145 As shown in Figure 4 (see additional results in Supplementary Information), under

146 MNAR missing mechanism assumption, SVT and Min/2 yielded the best performance

147 in both simulation settings, the relative performance of SVT and Min/2 depends on the

148 missing rates and criterion used for evaluation. The MNAR-devoted method, Min/2,

149 performs much better than the others as expected while the baseline method, Mean,

150 performance is the worst among all methods (see additional results in Supplementary

151 Information). Note that SOR increases expectedly when the missing rate increases

152 because SOR is positively associated with the number of missing proteins, therefore the

153 value of SOR may not imply the absolute performance of a method.

\section{Performance assessment focused on MCAR}

155 The imputation performance of the eight methods on MCAR mechanism is shown in

156 Figure 5 (see additional results in Supplementary Information). The experimental

157 results show that NIPALS outperforms all other methods in both simulation settings

158 and for almost all three evaluation criteria; while SVT is the best when RMSE is used;

$159 \mathrm{Min} / 2$ performs the worst among all other methods in all cases that may be expected

160 due to its design for MNAR mechanism; and Mean performs flatly over different

161 missing rates with an expected baseline performance except for Min/2. In addition,

162 while all methods perform worse when total missing rate increases, the ranking of their

163 relative performances remains unchanged (see additional results in Supplementary

164 Information). 


\section{Performance assessment focused on authentic missing values}

166 As shown in Figure 6 (see additional results in Supplementary Information), NIPALS,

167 SVT, and protein-wise or sample-wise KNN achieve the best performance, where

168 authentic missing values are dominant and imputation accuracy is evaluated on the

169 masked values. MNAR-devoted method Min/2 performs the worst as expected. Similar

170 to the case of MCAR, low-rank methods and local-similarity methods perform worse

171 when total missing rate increases, and among these methods, SVD and PPCA perform

172 even worse than the baseline method Mean when total missing rate is large. Note that

173 because low abundant proteins often have higher authentic missing rates and

174 accordingly higher masking rates, more low abundant proteins (possibly the minimum

175 values) are masked over highly expressed proteins, and the counterintuitive decrease in

176 NRMSE by Min/2 is expected when the authentic missing rate increases.

177 Evaluation of the FRMF method focused on authentic missing values

178 We evaluated three variants of the FRMF method. RMF serves as a baseline regularized

179 matrix factorization algorithm; FRMF_self introduces a fused-regularization utilizing

180 the similarity among samples embedded within data matrix; and FRMF_cross_patho

181 exploits external pathological scores again via fused-regularization strategy where the

182 pathological scores are the qualitative percentages of the intimal surface involvement

183 of various atherosclerotic changes graded by pathologists [6].

184 The experimental results are shown in Figure 7. While RMF performs

185 comparably and expectedly to SVD, both FRMF_self and FRMF_cross_patho

186 significantly outperform RMF. This preliminary result indicates a potential benefit of

187 combining global low-rank and local-similarly regularizations, as well as leveraging

188 external information via fused regularization. 
189

190

191

192

193

194

\section{Evaluation of the CAM method focused on authentic missing values}

Based on biologically-inspired latent variable modeling of complex tissues - CAM [13, 14], we proposed and evaluated three variants of the CAM based imputation method. CAM_complete performs CAM based imputation using the non-missing portion of full data matrix; CAM_SVT and CAM_NIPALS perform CAM based imputation using full data matrix while initialized by SVT and NIPALS, respectively.

The experimental results are shown in Figure 8. Expectedly, CAM_complete performs much better than the baseline method Mean. More importantly, both CAM_NIPALS and CAM_SVT consistently outperform NIPALS and SVT - the two top performers indicated in our earlier comparative assessment. This preliminary result suggests that biologically-plausible latent variable modeling may potentially improve imputation accuracy within the framework of low-rank optimization.

\section{Method}

\section{Brief introduction to the eight existing methods}

- Min/2 (half minimum): Assuming the MNAR missing mechanism, for each protein, replacing missing values with half the minimum value of observed intensities in that protein across samples $[6,10]$.

- Mean: Assuming the MAR/MCAR missing mechanism, for each protein, replacing missing values with mean value of observed intensities in that protein across samples $[6,10]$.

- $\quad$ swKNN (sample-wise k-nearest neighbors): Assuming the MAR missing mechanism and leveraging local similarity among samples, for each protein, replacing missing values with weighted average of observed intensities in that protein proportional to the proximities of k-nearest neighboring samples [10]. 
- pwKNN (protein-wise k-nearest neighbors): Assuming the MAR missing mechanism and leveraging local similarity among proteins, for each sample, replacing the missing values with weighted average of observed intensities in that sample proportional to the proximities of k-nearest neighboring proteins (with protein-wise normalization) [10].

- PPCA (probabilistic PCA): Assuming the MCAR/MAR missing mechanism,

a low-rank probabilistic PCA matrix factorization is estimated by the expectation maximization (EM) algorithm and subsequently used to impute missing values [23].

- NIPALS (non-linear estimation by iterative partial least squares): Assuming the MCAR/MAR missing mechanism, a low-rank missing-datatolerant PCA matrix factorization is estimated by iterative regression and subsequently used to impute missing values $[24,25]$.

- SVD (SVDImpute): Assuming the MCAR/MAR missing mechanism, a lowrank SVD matrix factorization is estimated by the EM algorithm and subsequently used to impute missing values [24, 26].

- SVT (singular value thresholding): Assuming MCAR/MAR missing mechanism, a low-rank SVT matrix factorization is estimated by iteratively solving a nuclear norm minimization problem and subsequently used to impute

\section{Performance measures}

Three quantitative measures are used to evaluate imputation accuracy, namely

235 Root Mean Square Error (RMSE), Normalized Root Mean Square Error (NRMSE), and

236 Sum of Ranks (SOR). Specifically, RMSE and NRMSE are given by [27, 28] 


$$
\operatorname{RMSE}=\sqrt{\frac{\sum_{\Omega}\left(\hat{X}_{\Omega}-X_{\Omega}\right)^{2}}{|\Omega|}}, \quad \text { NRMSE }=\sqrt{\frac{\sum_{\Omega}\left(\hat{X}_{\Omega}-X_{\Omega}\right)^{2}}{|\Omega| \sigma_{X_{\Omega}}^{2}}}
$$

238 respectively, where $\Omega$ is the index set of missing values in complete data matrix $X,|\Omega|$

239 is the total number of missing values, $\hat{X}$ is the imputed complete data matrix, and $\sigma_{X_{\Omega}}^{2}$

240 is the variance of missing values. To address the bias of NRMSE under MNAR missing 241 mechanism, SOR has been proposed as [19]

$$
\mathrm{SOR}=\sum_{i=1}^{P} \operatorname{rank}\left(\mathrm{NRMSE}_{i}\right),
$$

243 where $P$ is the number of proteins containing at least one missing value, $i$ is the protein 244 index in this protein subset, and $\operatorname{rank}\left(\mathrm{NRMSE}_{i}\right)$ is the ranks of protein-wise NRMSE 245 across different imputation methods.

\section{Introduction to FRMF method}

247 As aforementioned, low-rank matrix factorization has been a popular and effective 248 approach for missing data imputation [12]. For imputing proteomics data, the 249 assumption is that there is only a small number of biological processes determining the 250 expression profiles. Consider an $m \times n$ complete data matrix $X$ describing $m$ samples 251 and $n$ proteins. A low-rank matrix factorization approach seeks to approximate $X$ 252 containing missing values by a linear latent variable model,

$$
X_{m \times n}=A_{m \times l} \times S_{l \times n},
$$

254 where $A_{m \times l}$ and $S_{l \times n}$ are the low-rank factor matrices, and $l \ll \min (m, n)$. In order to 255 prevent overfitting, the solution is often formulated as a regularized sparse SVD 256 minimization problem on the observed values

$$
\min \sum_{i=1}^{m} \sum_{j=1}^{n} I\left(X_{i j} \neq \mathrm{NA}\right)\left(X_{i j}-A_{i} S_{j}\right)^{2}+\lambda_{\mathrm{A}}\|A\|_{F}^{2}+\lambda_{\mathrm{S}}\|S\|_{F}^{2},
$$


where $\|.\|_{F}^{2}$ denotes the Frobenius norm, $I($.$) is the indicator function, and \lambda_{\mathrm{A}}, \lambda_{\mathrm{S}}>0$ are the regularization parameters. When local similarity information is available, FRMF can be formulated by adding a fused regularization term

$$
\begin{aligned}
& \min \sum_{i=1}^{m} \sum_{j=1}^{n} I\left(X_{i j} \neq \mathrm{NA}\right)\left(X_{i j}-A_{i} S_{j}\right)^{2}+\lambda_{\mathrm{A}}\|A\|_{F}^{2}+\lambda_{\mathrm{S}}\|S\|_{F}^{2} \\
& +\alpha \sum_{i=1}^{m} \sum_{k \in \mathcal{F}(i)}\left\|A_{i}-A_{k}\right\|_{F}^{2}
\end{aligned}
$$

where $\alpha$ is the fused regularization parameter, and $\mathcal{F}(i)$ denotes the neighborhood sample subset of sample $i$ and can be determined using baseline data or other relevant measurements e.g. gene expression or pathological score. In our study, $\mathcal{F}(i)$ is determined by the between-sample cosine similarity $\cos \left(X_{i}, X_{k}\right)$ based on data matrix in FRMF_self, or $\cos \left(P_{i}, P_{k}\right)$ based on pathological scores in FRMF_cross_patho.

\section{Introduction to CAM method}

CAM is a latent variable modeling and deconvolution technique previously used for identifying biologically-interpretable cell subtypes $S_{l \times n}$ and their composition $A_{m \times l}$ in complex tissues $[6,13,14,21]$. We adopt the CAM framework into (1) and demonstrate that hybrid CAM_SVT and CAM_NIPALS can handle missing values naturally and this combination leads to a novel and biologically-plausible imputation strategy. The workflow of CAM based method with three variants is given in Figure 9.

\section{Discussion}

The quality of simulating assumed missing mechanisms (MNAR and MCAR) depends on the efficacy of simulation tools. However, because the simulation uses only the observed portion of data matrix, the introduced artificial missing values cannot fully resemble authentic missing mechanisms and/or patterns in relation to original overall 
280 data distribution. More critically, performance is actually assessed on imputing 281 artificial not authentic missing values, where the overall data distribution may be 282 distorted.

To address the aforementioned issues in the presence of authentic missing 284 values, a small percentage of set-aside values are introduced into complete data matrix 285 and used solely for assessment purpose. Because masked values are randomly assigned 286 onto both observed and authentic missing values, the simulation maximally preserves 287 original overall data distribution. Note that masked values may represent a mix of 288 MNAR (high missing rate associated with low protein abundance) and MAR (joint 289 distribution of both observed and authentic missing values). However, performance is 290 assessed indirectly on imputing masked not authentic missing values. Imputation accuracy would be arguably affected by data preprocessing and 292 algorithmic parameter setting. In this study, sample-wise normalization and protein293 wise standardization are performed based on the requirements of each method. While 294 these preprocessing notably affects the scale of NRMSE, relative performances across 295 methods remain consistent. The experimental results show that imputation performance 296 varies with parameter setting, while there appears no theoretical guideline for 297 optimizing parameter setting.

298 While FRMF is a promising and novel imputation approach, its effectiveness 299 for improving classic low-rank methods would depend on diversity among samples, 300 discriminatory power of similarity measure, and complementary nature of additional 301 and relevant measurements. Newly proposed CAM method represents an interesting 302 direction for further development. More importantly, CAM performs missing value 303 imputation using original intensity rather than log-transformed data, and this is 
304 mathematically more rigorous because log-transformation violates the linear nature of

305 low-rank matrix factorization [29].

\section{Declarations}

308 - $\quad$ Ethics approval and consent to participate

$309 \quad$ Not applicable

$310 \quad$ - $\quad$ Consent for publication

$311 \quad$ Not applicable

$312 \quad$ - $\quad$ Availability of data and materials

313 The scripts used in the paper is available in R script ProImput.

314 Code for all experiments can be found in the vignette at

315 https://github.com/MinjieSh/ProImput. The operation system

$316 \quad$ can be any system supporting $\mathrm{R}$ language.

$317 \quad$ - Competing interests

318 The authors declare that they have no competing interests.

319 - Funding

320 This work has been supported by the National Institutes of 321 Health under Grants HL111362-05A1, HL133932, NS11565832201 , and the Department of Defense under Grant W81XWH-18$323 \quad 1-0723(\mathrm{BC} 171885 \mathrm{P} 1)$.

324 - $\quad$ Authors' Contributions

325 MJS, YTC, CTW and YW initiated this research and developed 326 the methods. MJS and YW drafted the manuscript. MJS and 327 CTW developed the assessment procedure and software. SJP 
and GS contributed with biomedical case study. YZW, GQY, JEVE and DMH contributed with biomedical significance and discussion of the work. All authors have read, commented on and accepted the final manuscript.

Not applicable

\section{References}

1. Canterbury JD, Merrihew GE, MacCoss MJ, Goodlett DR, Shaffer SA: Comparison of data acquisition strategies on quadrupole ion trap instrumentation for shotgun proteomics. Journal of The American Society for Mass Spectrometry 2014, 25(12):2048-2059.

2. Doerr A: DIA mass spectrometry. Nature methods 2014, 12(1):35.

3. Goeminne LJE, Sticker A, Martens L, Gevaert K, Clement L: MSqRob Takes the Missing Hurdle: Uniting Intensity- and Count-Based Proteomics. Anal Chem 2020, 92(9):6278-6287.

4. Ma W, al. e, Wang P: DreamAI: algorithm for the imputation of proteomics data. bioRxiv 2020.

5. Dabke K, Kreimer S, Jones MR, Parker SJ: A Simple Optimization Workflow to Enable Precise and Accurate Imputation of Missing Values in Proteomic Datasets. bioRxiv 2020.

6. Herrington DM, Mao C, Parker SJ, Fu Z, Yu G, Chen L, Venkatraman V, Fu Y, Wang Y, Howard TD et al: Proteomic Architecture of Human Coronary and Aortic Atherosclerosis. Circulation 2018, 137(25):2741-2756.

7. Lazar C, Gatto L, Ferro M, Bruley C, Burger T: Accounting for the multiple natures of missing values in label-free quantitative proteomics data sets to compare imputation strategies. Journal of proteome research 2016, 15(4):1116-1125.

8. Jakobsen JC, Gluud C, Wetterslev J, Winkel P: When and how should multiple imputation be used for handling missing data in randomised clinical trials - a practical guide with flowcharts. BMC Med Res Methodol 2017, 17(1):162.

9. Webb-Robertson B-JM, Wiberg HK, Matzke MM, Brown JN, Wang J, McDermott JE, Smith RD, Rodland KD, Metz TO, Pounds JG: Review, evaluation, and discussion of the challenges of missing value imputation for mass spectrometry-based label-free global proteomics. Journal of proteome research 2015, 14(5):1993-2001.

10. Liu M, Dongre A: Proper imputation of missing values in proteomics datasets for differential expression analysis. Brief Bioinform 2020.

11. Lin X, Boutros PC: Optimization and expansion of non-negative matrix factorization. BMC bioinformatics 2020, 21(1):7. 
12. Ma H, Zhou D, Liu C, Lyu MR, King I: Recommender systems with social regularization. In: The fourth ACM international conference on Web search and data mining: 2011; Hong Kong. ACM Press: 287-296.

13. Wang N, Hoffman EP, Chen L, Chen L, Zhang Z, Liu C, Yu G, Herrington DM, Clarke R, Wang Y: Mathematical modelling of transcriptional heterogeneity identifies novel markers and subpopulations in complex tissues. Scientific Reports 2016, 6:18909.

14. Chen L, Wu CT, Wang N, Herrington DM, Clarke R, Wang Y: debCAM: a bioconductor $\mathrm{R}$ package for fully unsupervised deconvolution of complex tissues. Bioinformatics (Oxford, England) 2020, 36(12):3927-3929.

15. Rahman SA, Huang Y, Claassen J, Heintzman N, Kleinberg S: Combining Fourier and lagged k-nearest neighbor imputation for biomedical time series data. Journal of biomedical informatics 2015, 58:198-207.

16. Pedersen AB, Mikkelsen EM, Cronin-Fenton D, Kristensen NR, Pham TM, Pedersen L, Petersen I: Missing data and multiple imputation in clinical epidemiological research. Clinical epidemiology 2017, 9:157.

17. John C, Ekpenyong EJ, Nworu CC: Imputation of missing values in economic and financial time series data using five principal component analysis approaches. CBN Journal of Applied Statistics 2019, 10(1):51-73.

18. Cai J-F, Candès EJ, Shen Z: A singular value thresholding algorithm for matrix completion. SIAM Journal on optimization 2010, 20(4):1956-1982.

19. Wei R, Wang J, Su M, Jia E, Chen S, Chen T, Ni Y: Missing value imputation approach for mass spectrometry-based metabolomics data. Scientific reports 2018, 8(1):1-10.

20. Teo G, Kim S, Tsou C-C, Collins B, Gingras A-C, Nesvizhskii AI, Choi H: mapDIA: Preprocessing and statistical analysis of quantitative proteomics data from data independent acquisition mass spectrometry. Journal of proteomics 2015, 129:108-120.

21. Parker SJ, Chen L, Spivia W, Saylor G, Mao C, Venkatraman V, Holewinski RJ, Mastali M, Pandey R, Athas G et al: Identification of Putative Early Atherosclerosis Biomarkers by Unsupervised Deconvolution of Heterogeneous Vascular Proteomes. J Proteome Res 2020, 19(7):27942806.

22. imputeLCMD: A collection of methods for left-censored missing data imputation [https://cran.r-project.org/package=imputeLCMD]

23. Tipping ME, Bishop CM: Probabilistic principal component analysis. Journal of the Royal Statistical Society: Series B (Statistical Methodology) 1999, 61(3):611-622.

24. Stacklies W, Redestig H, Scholz M, Walther D, Selbig J: pcaMethods-a bioconductor package providing PCA methods for incomplete data. Bioinformatics 2007, 23(9):1164-1167.

25. Ochoa-Muñoz AF, González-Rojas VM, Pardo CE: Missing data in multiple correspondence analysis under the available data principle of the NIPALS algorithm. DYNA 2019, 86(211):249-257.

26. Troyanskaya O, Cantor M, Sherlock G, Brown P, Hastie T, Tibshirani R, Botstein D, Altman RB: Missing value estimation methods for DNA microarrays. Bioinformatics 2001, 17(6):520-525.

27. Stekhoven DJ, Bühlmann P: MissForest—non-parametric missing value imputation for mixed-type data. Bioinformatics 2012, 28(1):112-118. 
28. Oba S, Sato M, Takemasa I, Monden M, Matsubara K, Ishii S: A Bayesian missing value estimation method for gene expression profile data. Bioinformatics 2003, 19(16):2088-2096.

29. Zhong Y, Liu Z: Gene expression deconvolution in linear space. Nat Methods 2011, 9(1):8-9; author reply 9. 


\section{Figures}

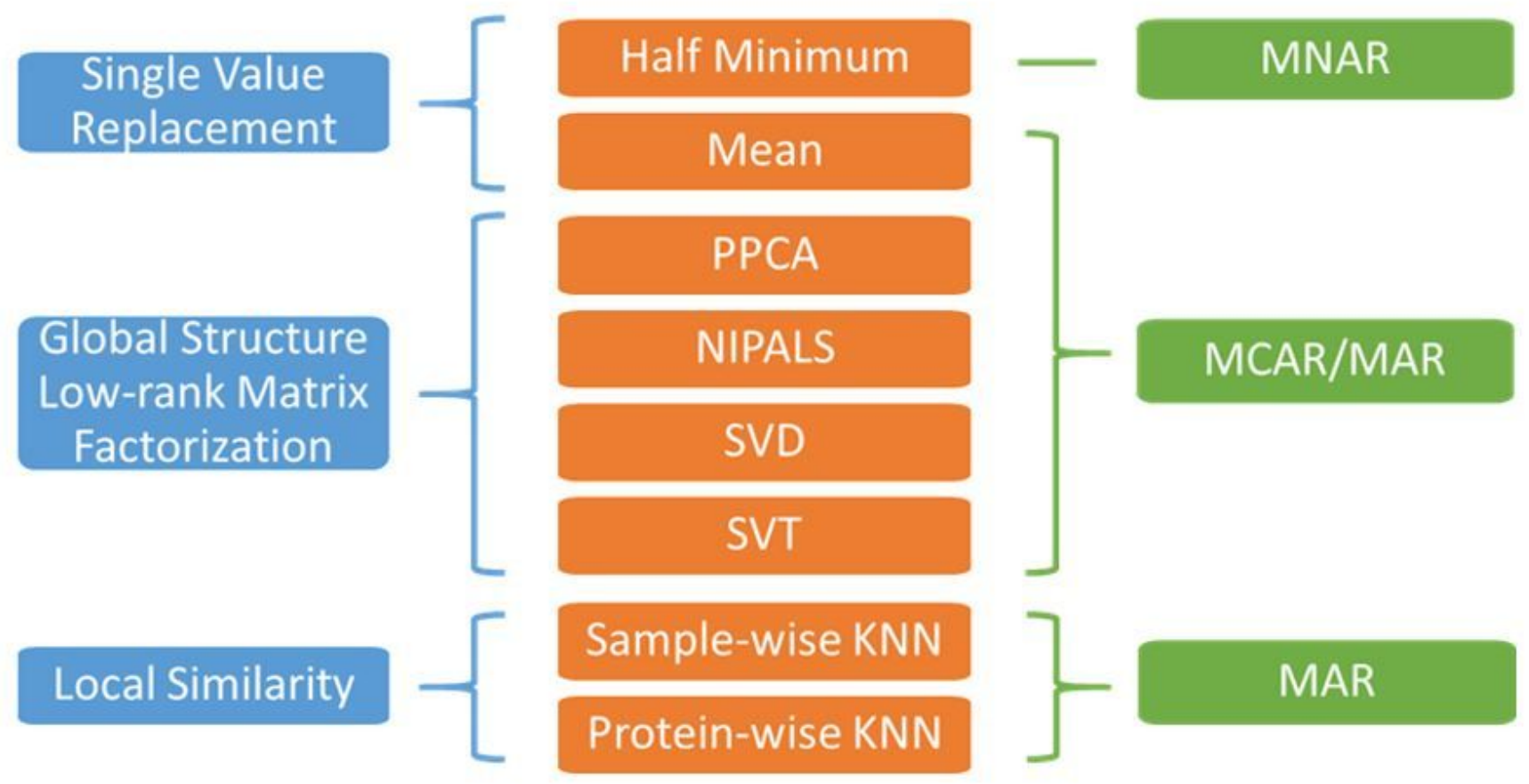

\section{Figure 1}

Comparative assessment of eight representative missing value imputation methods, divided into three categories.

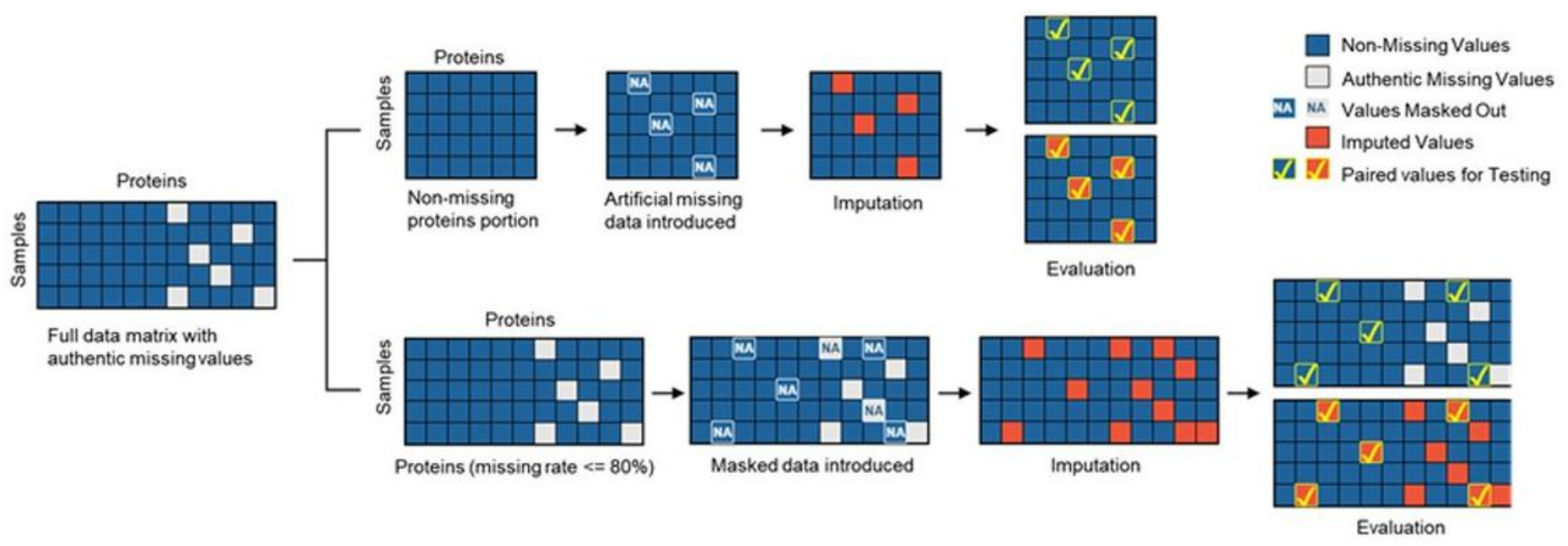

\section{Figure 2}

Two-phased workflow of realistic simulation-based assessment on missing value imputation methods. 
Original full data matrix

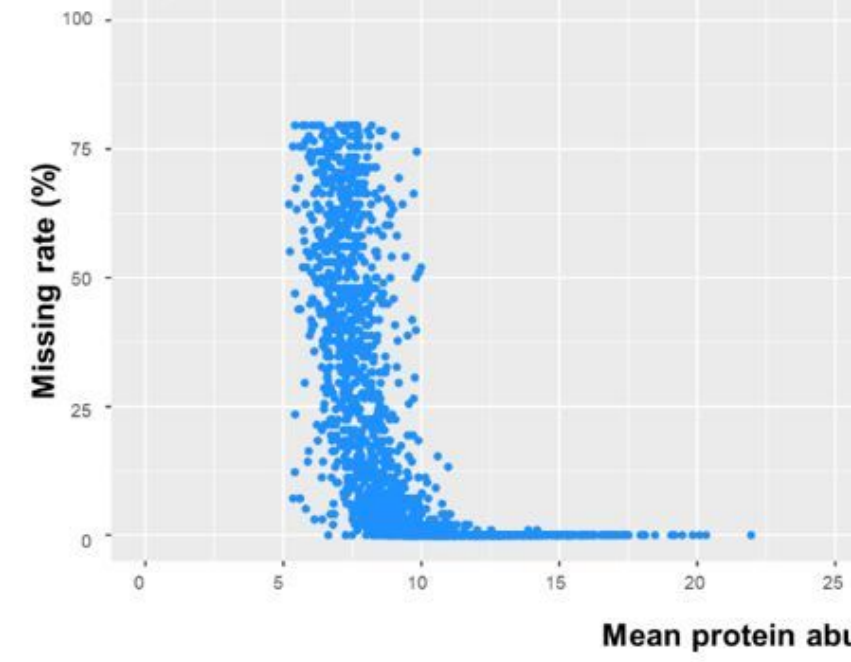

Full data matrix with masked NA

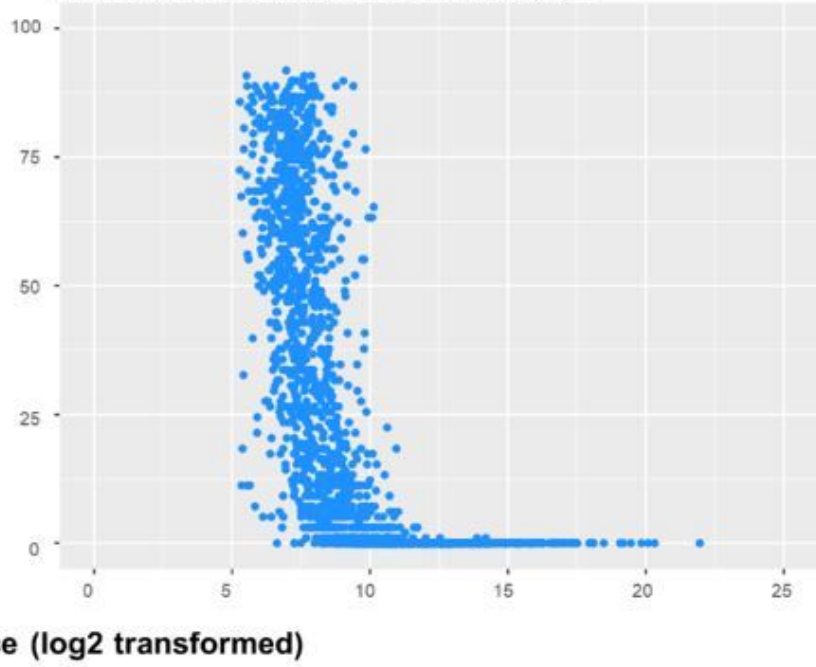

Figure 3

The overall pattern of missing values illustrated by the relationship between protein missing rate and protein mean intensity, before (left panel) and after (right panel) introducing masked NA.

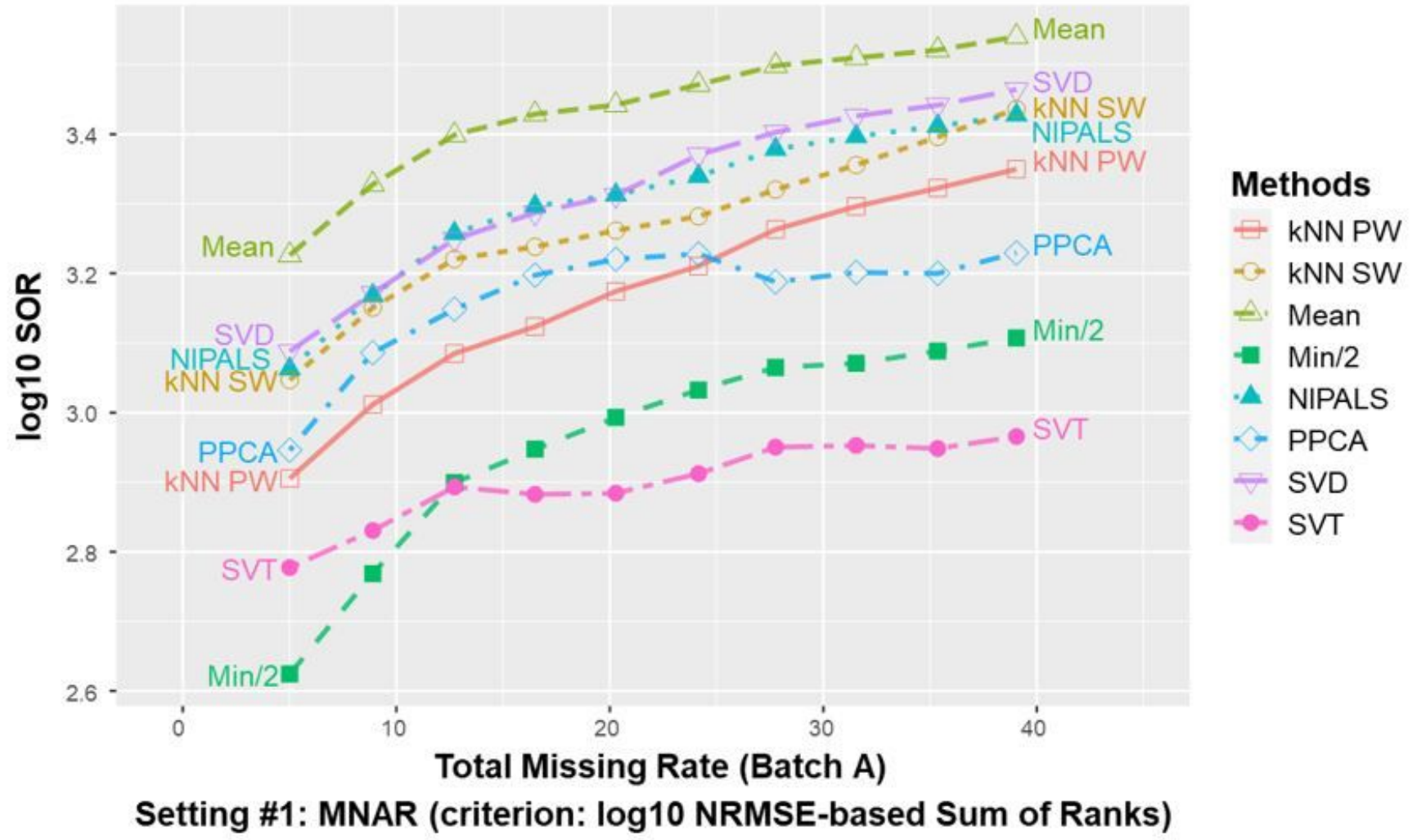

Figure 4 
Imputation performance of the eight methods on the simulation data of setting \#1, with assumed MNAR missing mechanism and varying total missing rates.

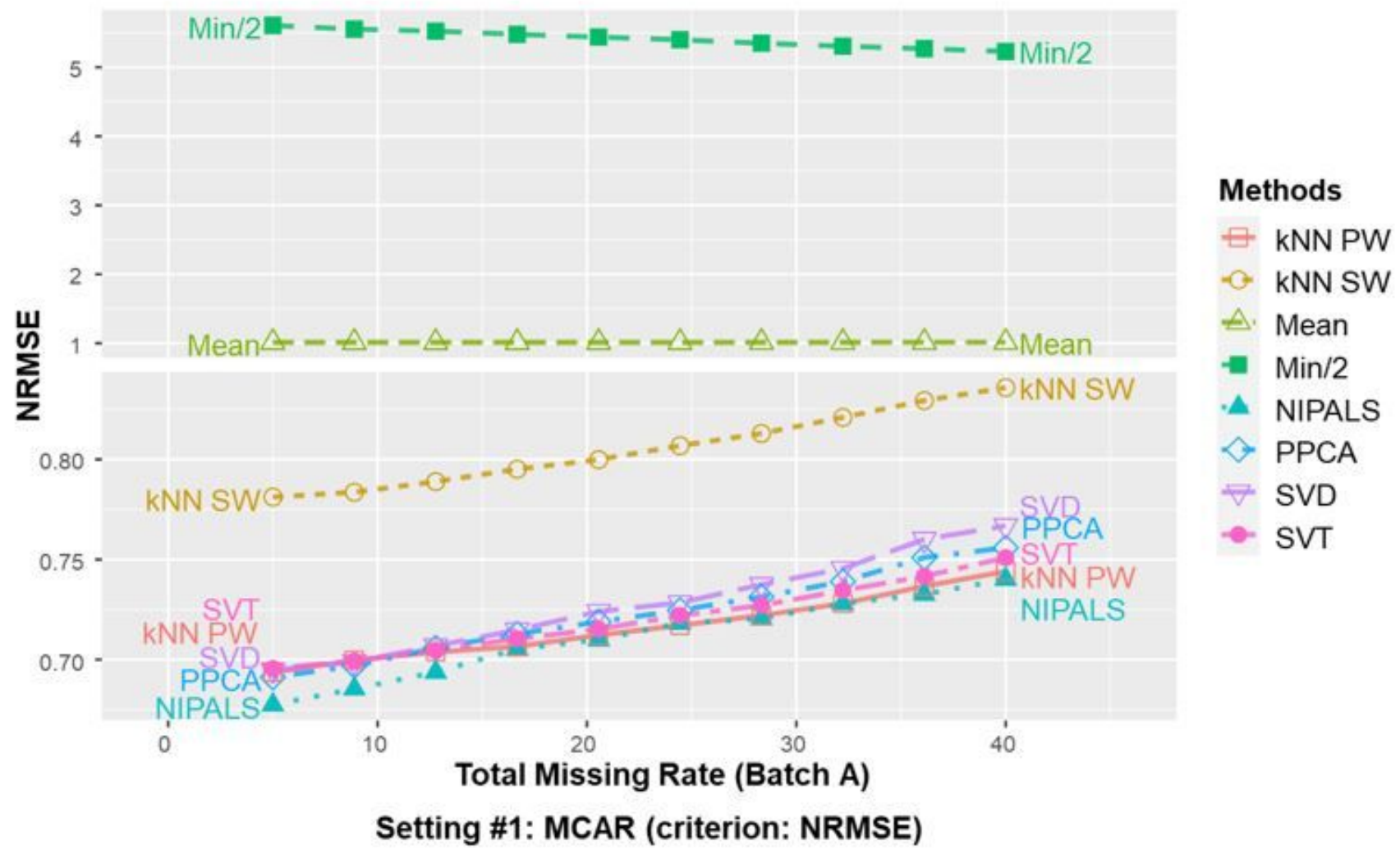

\section{Figure 5}

Imputation performance of the eight methods on the simulation data of setting \#1, with assumed MCAR missing mechanism and varying total missing rates. 


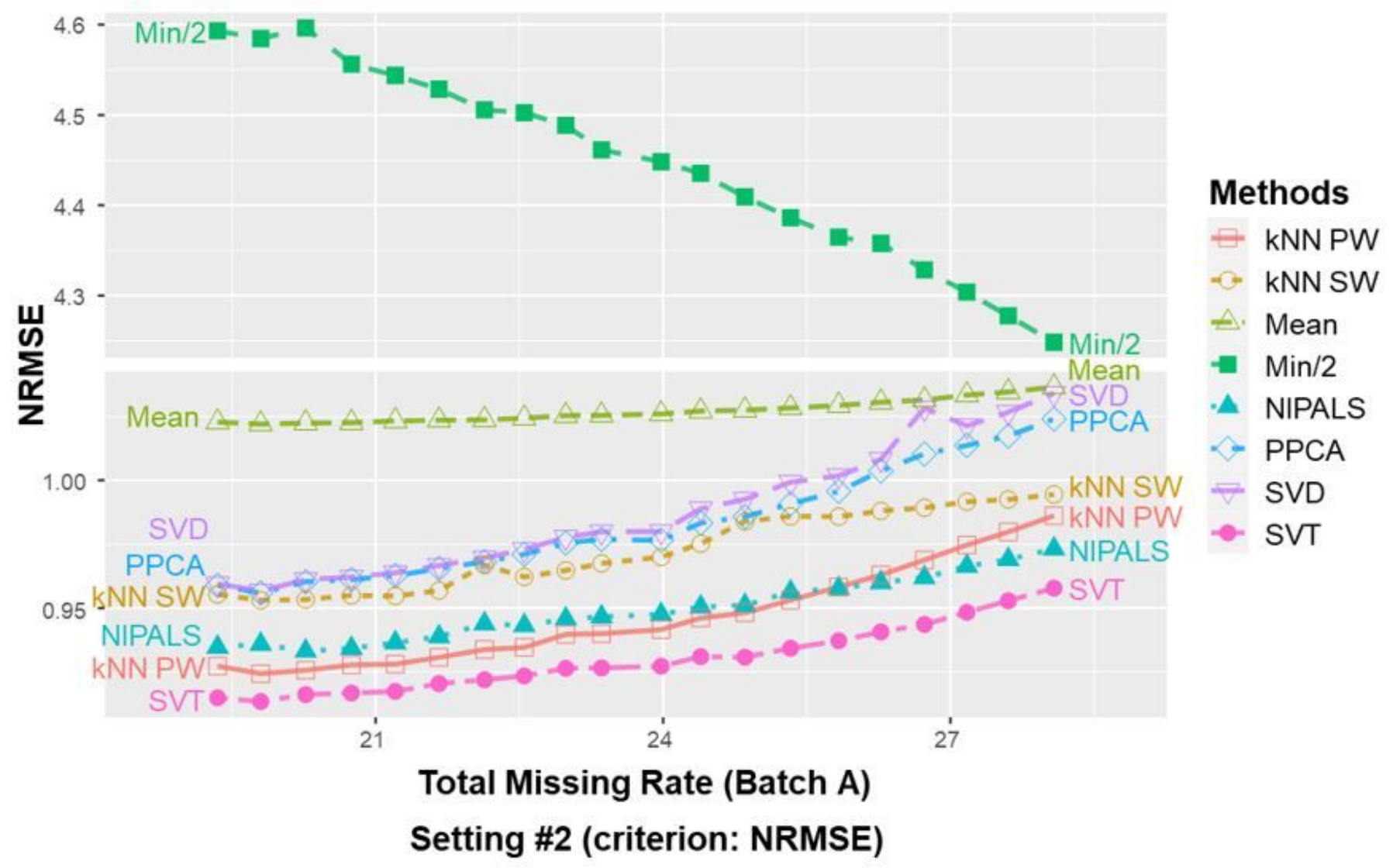

Figure 6

Imputation performance of the eight methods on the simulation data of setting \#2, focusing on authentic missing mechanism and varying masked rates. 


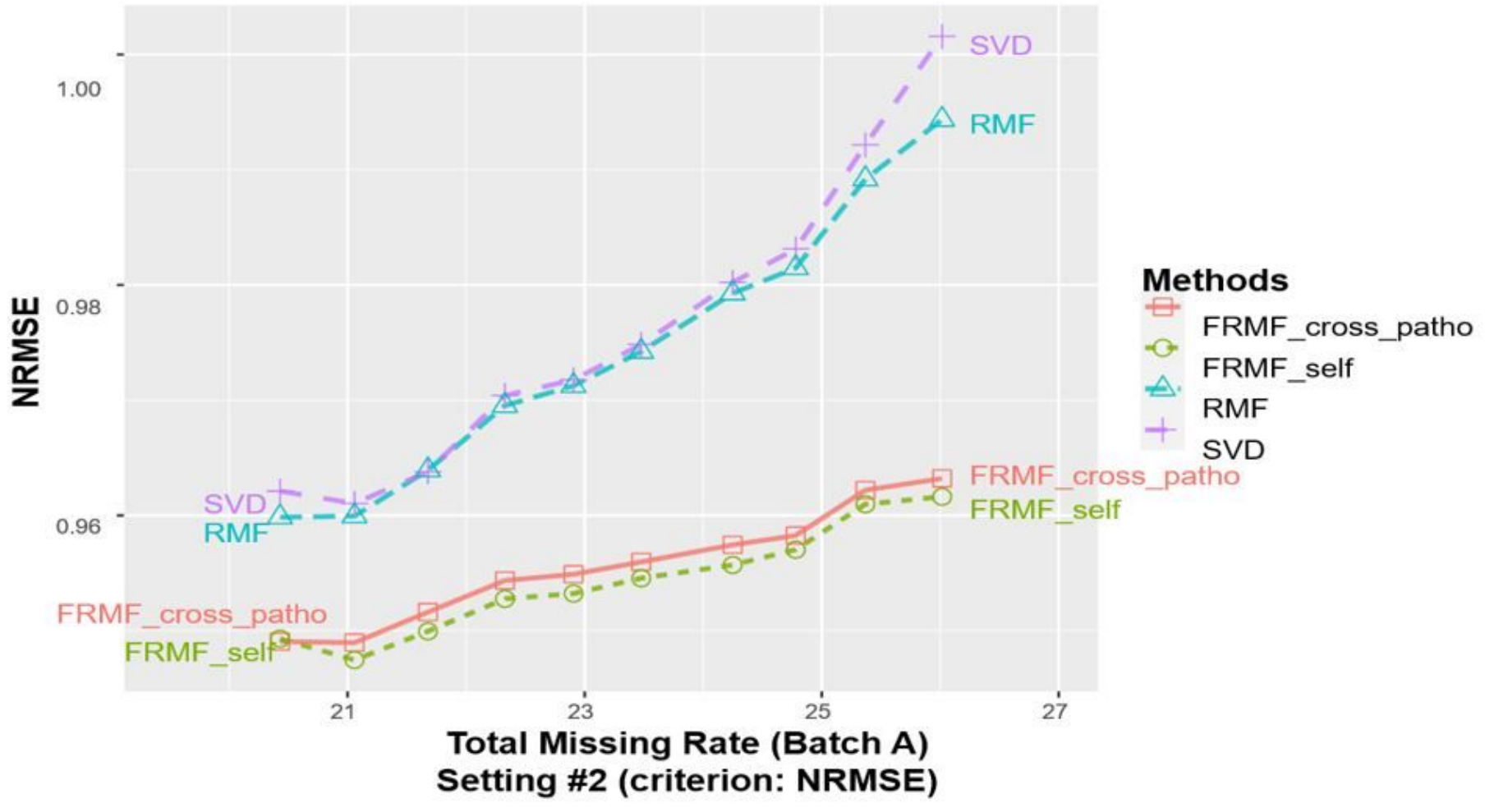

Figure 7

Imputation performance of the FRMF variants on the simulation data of setting \#2, with varying masked rates.

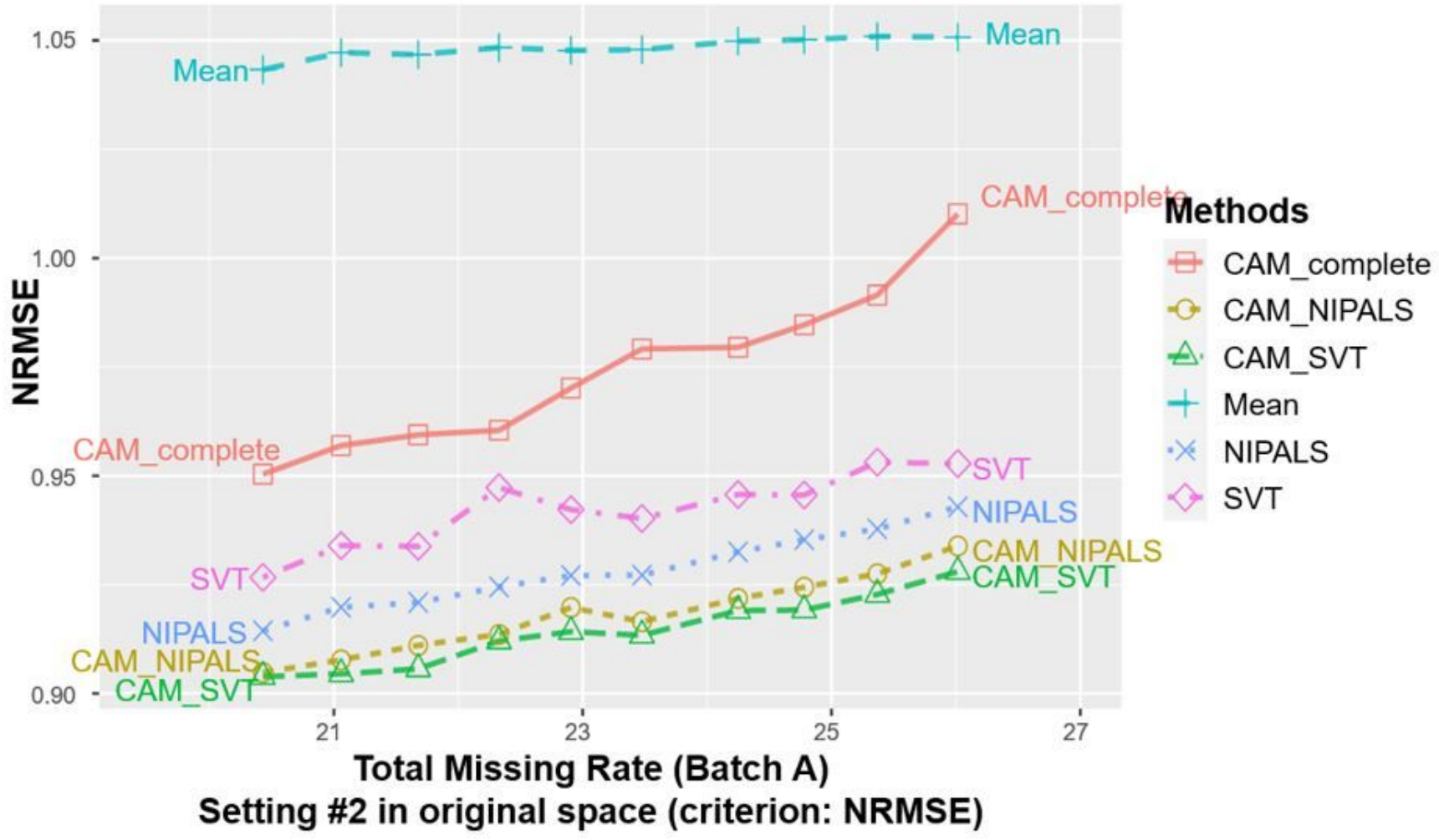




\section{Figure 8}

Imputation performance of CAM variants on the simulation data of setting \#2, with varying masked rates, in comparison to that of Mean, SVT, and NIPALS. The imputation accuracy is evaluated in the original intensity space (before log-transformation).

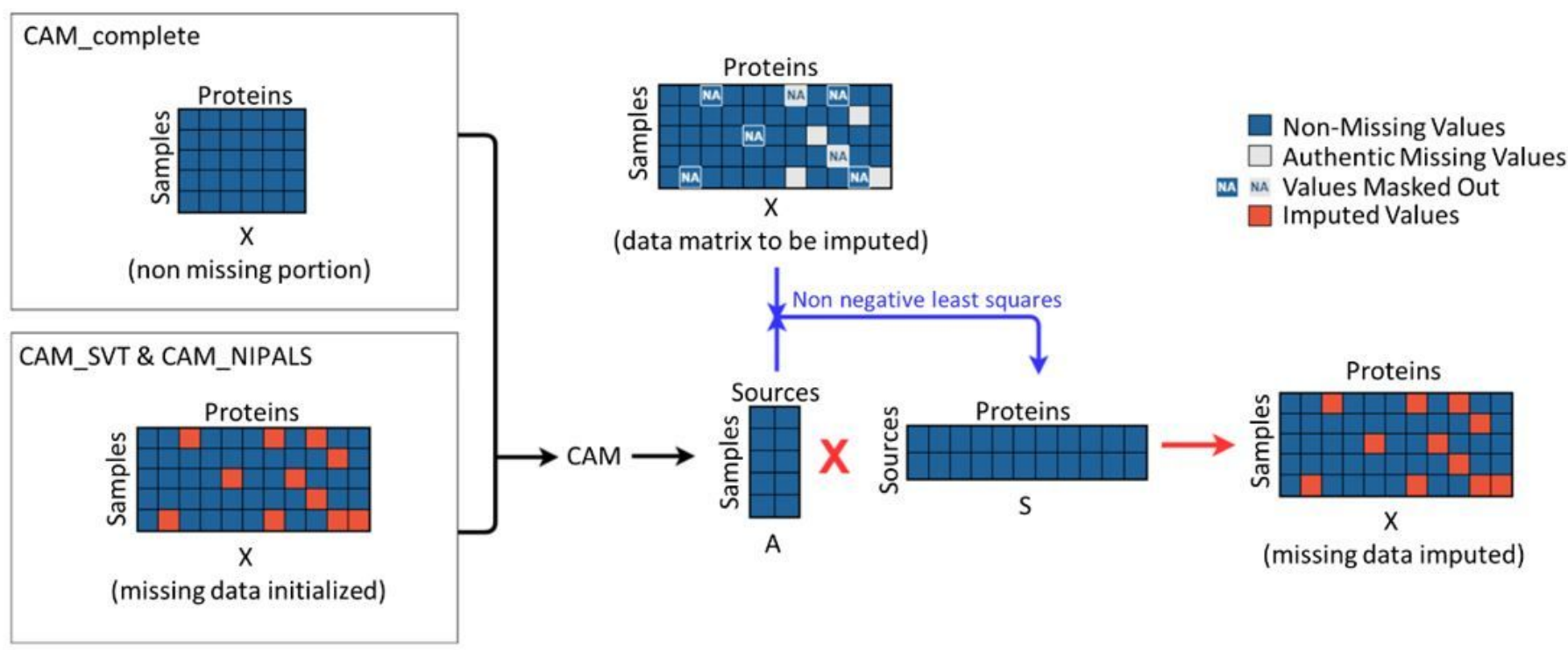

Figure 9

Workflow of the CAM based imputation method with two variant algorithms.

\section{Supplementary Files}

This is a list of supplementary files associated with this preprint. Click to download.

- Supplementarylnformation.docx 